\title{
In situ TEM Measurements of Ion Irradiation Induced Creep
}

\author{
Gowtham S. Jawaharram ${ }^{1}$, Christopher Barr ${ }^{2}$, Patrick Price ${ }^{2}$, Khalid Hattar ${ }^{2}$ and Shen J. Dillon ${ }^{1 *}$
}

1. Department of Materials Science and Engineering, University of Illinois Urbana-Champaign, Urbana, IL, USA.

2. Sandia National Laboratories, Albuquerque, NM, USA.

* Corresponding author: sdillon@illinois.edu

Novel alloys and materials have been developed for next generation reactors and systems exposed to irradiation. Great emphasis has been placed on nanostructured materials that have high densities of unsaturable sinks to facilitate efficient point defect recombination, which is critical for suppressing a variety of irradiation induced damage. Additionally, nanostructured materials often exhibit exceptional mechanical properties making them well suited for structural applications in extreme environments. However, little is known about their thermomechanical response under irradiation. The paucity of data describing deformation under irradiation and IIC derives from challenges associated with experimental measurements, which may be performed with neutrons, ions, or electrons. Irradiation creep measurements in reactors typically require long experiments (e.g. months), are expensive, require large test specimens, and can result in radioactive waste $[1,2]$. High energy light ions and electrons can be used to test samples at somewhat accelerated rates, but their recoil spectra differ from neutrons, and energy dissipation proceeds primarily via electronic stopping resulting in low dpa rates at reasonable fluxes [3-5]. High energy heavy ions simulate neutrons reasonably well and can achieve 10's to 100's of dpa on the order of 1 day, but their average penetration range is typically on the order of 100's of nanometers. As a result, there is potential for accelerated IIC experiments using high energy heavy ion irradiation, provided that the experiments are performed on nanoscale specimens [6-9]. Such small specimens present a variety of challenges when tested ex situ [6, 7]. In situ transmission electron microscopy is ideal for manipulating and testing nanoscale specimens and observing their deformation in real time to ensure the appropriate deformation modes are active. We previously developed such an approach to performing in situ irradiation induced creep in a transmission electron microscope $[10,11]$. The first iteration demonstrated that it was possible to measure room temperature irradiation induced creep in nanolaminates [10]. The second iteration demonstrated that laser heating could be used to effectively measure irradiation induced compressive creep at high temperatures in Ag pillars [11]. In that experiment, the temperature was determined somewhat indirectly. A variety of methods may be employed for temperature determination in the TEM; such as via shift in the plasmon peak or lattice parameter changes measured by electron diffraction. In these experiments a $\approx 100 \mu \mathrm{m}$ diameter, $\lambda=$ $1064 \mathrm{~nm}$ laser is used to heat samples mounted on a Hysitron PI-95 picoindenter. An example plot of applied laser power versus temperature determined from thermal expansion measured from $\mathrm{Cu}$ nanopillars on a $\mathrm{Y}_{2} \mathrm{O}_{3}$-stabilized $\mathrm{ZrO}_{2}$ substrate is shown in Figure 1. The results demonstrate that samples may be heated to high temperatures, $\mathrm{T}>1000^{\circ} \mathrm{C}$, at modest laser powers. The results suggest that it is possible to mechanically test samples over a broad range of temperatures using in situ TEM combined with laser heating. When additionally combined with in situ ion irradiation it is possible to characterize irradiation induced creep, and related mechanical properties, under a variety of conditions. This presentation will discuss application of this technique to other alloy systems such as high entropy alloys, Ag alloys, and $\mathrm{Al}$ alloys [12]. 
References:

[1] FA Garner, MB Toloczko and BH Sencer, J. Nucl. Mater. 276 (2000), p. 123.

[2] BT Kelly and JE Brocklehurst, J. Nucl. Mater. 65(1) (1977), p. 79.

[3] J Chen et al., J. Nucl. Mater. 386-388 (2009), p. 143.

[4] P Jung, Journal of Nuclear Materials. 200(1) (1993), p. 138.

[5] C Xu and GS Was, J. Nucl. Mater. 459 (2015), p. 183.

[6] S OzerInc, RS Averback and WP King, JOM. 68(11) (2016), p. 2737.

[7] S Ozerinc et al., J. Appl. Phys.. 117(2) (2015), p. 024310/1.

[8] K Tai et al., J. Nucl. Mater. 422 (2012), p. 8.

[9] P Lapouge et al., J. Nucl. Mater. 476 (2016), p. 20.

[10] SJ Dillon et al., J. Nucl. Mater. 490 (2017), p. 59.

[11] GS Jawaharram et al., Scr. Mater. 148 (2018), p. 1.

[12] This work was performed, in part, at the Center for Integrated Nanotechnologies, an Office of Science User Facility operated for the U.S. Department of Energy (DOE) Office of Science. Sandia National Laboratories is a multi-mission laboratory managed and operated by National Technology and Engineering Solutions of Sandia, LLC., a wholly owned subsidiary of Honeywell International, Inc., for the U.S. DOE's National Nuclear Security Administration under contract DE-NA-0003525. The views expressed in the article do not necessarily represent the views of the U.S. DOE or the United States Government. G.J. and S.J.D. would like to acknowledge US DOE Office of Basic Energy Sciences, under Grant DEFG02-05ER46217.
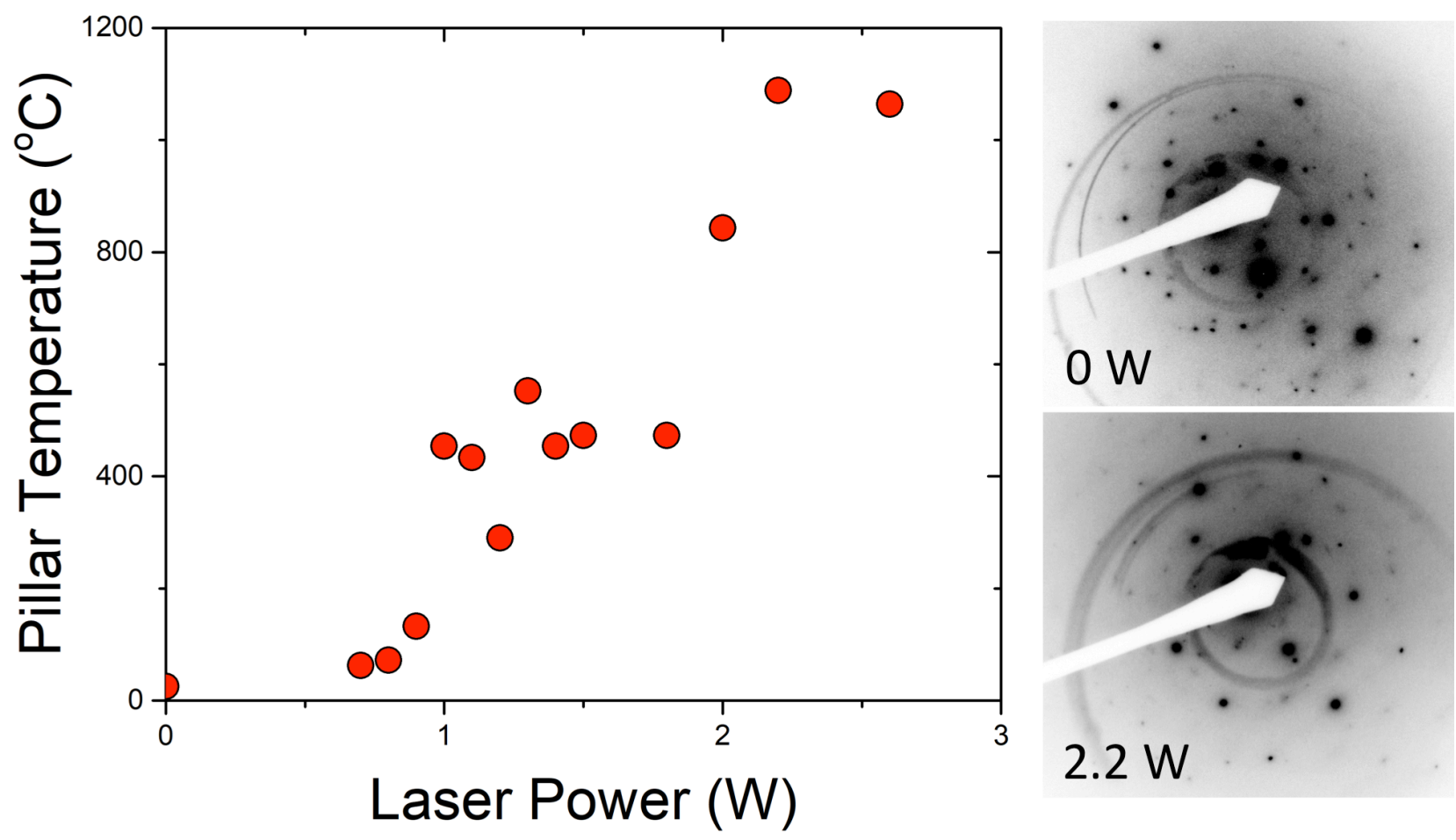

Figure 1. Plot of pillar temperature determined from electron diffraction versus applied laser power for a $\mathrm{Cu}$ pillar grown on a single crystal $\mathrm{Y}_{2} \mathrm{O}_{3}$-stabilized $\mathrm{ZrO}_{2}$ substrate. The disappearance of diffraction spots occurs due to grain growth. 